\title{
A vectorlike representation of multilayers
}

\author{
Alberto G. Barriuso, Juan J. Monzón, and Luis L. Sánchez-Soto \\ Departamento de Óptica, Facultad de Física, Universidad Complutense, 28040 Madrid, Spain \\ José F. Cariñena \\ Departamento de Física Teórica, Facultad de Ciencias, \\ Universidad de Zaragoza, 50009 Zaragoza, Spain
}

\begin{abstract}
We resort to the concept of turns to provide a geometrical representation of the action of any lossless multilayer, which can be considered as the analogous in the unit disk to the sliding vectors in Euclidean geometry. This construction clearly shows the peculiar effects arising in the composition of multilayers. A simple optical experiment revealing the appearance of the Wigner angle is analyzed in this framework.

PACS numbers: 230.4170 Multilayers, 120.5700 Reflection, 120.7000 Transmission, 000.3860 Mathematical methods in physics
\end{abstract}

\section{INTRODUCTION}

The search for mathematical entities that could describe physical phenomena has always been a top priority. For example, the need to describe a direction in space, combined with the use of geometry to approach physical problems, brought forth the concept of a vector.

The idea that the complex numbers have a geometrical interpretation as vectors lying in a plane, led to Hamilton to introduce quaternions with the aim of being useful for the analysis of three-dimensional space [1]. The price to be paid is that the composition of quaternions is not commutative. Soon after that, it became clear that rotations can be advantageously represented by unit quaternions.

A notion closely related to Hamilton treatment is that of turns [2]. The turn associated with a rotation of axis $\hat{\mathbf{n}}$ and angle $\vartheta$ is a directed arc of length $\vartheta / 2$ on the great circle orthogonal to $\hat{\mathbf{n}}$ on the unit sphere. By means of these objects, the composition of rotations is described through a parallelogramlike law: if these turns are translated on the great circles, until the head of the arc of the first rotation coincides with the tail of the arc of the second one, then the turn between the free tail and the head is associated with the resultant rotation. Hamilton turns are thus analogous for spherical geometry to the sliding vectors in Euclidean geometry.

In recent years many concepts of geometrical nature have been introduced to gain further insights into the behavior of layered media. The algebraic basis for these developments is the fact that the transfer matrix associated with a lossless multilayer is an element of the group $\mathrm{SU}(1,1)$, which is locally isomorphic to the Lorentz group $\mathrm{SO}(2,1)$ in $(2+1)$ dimensions. This leads to a natural and complete identification between reflection and transmission coefficients and the parameters of the corresponding Lorentz transformation [3, 4].

In an appealing paper, Juárez and Santander [5] developed a generalization of Hamilton turns to the Lorentz group, while Simon, Mukunda, and Sudarshan [6, 7] worked out an equivalent algebraic approach for $\mathrm{SU}(1,1)$, in which they introduce a noncommutative geometrical addition for these hyperbolic turns that reproduces the composition law of the group; i. e., for both the reflection and transmission coefficients. The latter coefficients seem to be (almost) ignored in the literature. The goal of this paper is precisely to show how this formalism affords a very intuitive image of multilayer optics.

Of course, since the amplitude and phase of a light beam can be conveniently represented by a (Euclidean) vector, the properties of layered media have been represented by graphical constructions. These visualization tools are commonly in use in thin-film design and include the Smith chart, the admittance diagram 8], the (reflectance) circle diagrams [9], and the vector method [10], among others. However, the formalism of turns is intrinsic and does not rely on any specific representation for the light waves. In other words, once the turn for a multilayer is known, his action on any light state is fully determined. We emphasize that, although, these techniques can be used for quantitative calculations, they cannot compete nowadays with modern software design, and their great value is in the visualization of the characteristics of a multilayer.

This paper is organized as follows: In Section 2 we present some details of how the action of any multilayer can be seen as a geometrical motion in the unit disk. Every one of these motions can be decomposed in terms of two reflections, which justifies the idea of turn introduced in Section 3, where their composition law is also introduced via a parallelogram law, in close analogy with what happens for sliding vectors in Euclidean geometry. The noncommutative character of this law leads to interesting phenomena, such as the appearance of extra phases in the composition of multilayers, which is examined in Section 4, giving a simple though nontrivial example that illustrates clearly how this geometrical scenario works in practice. 


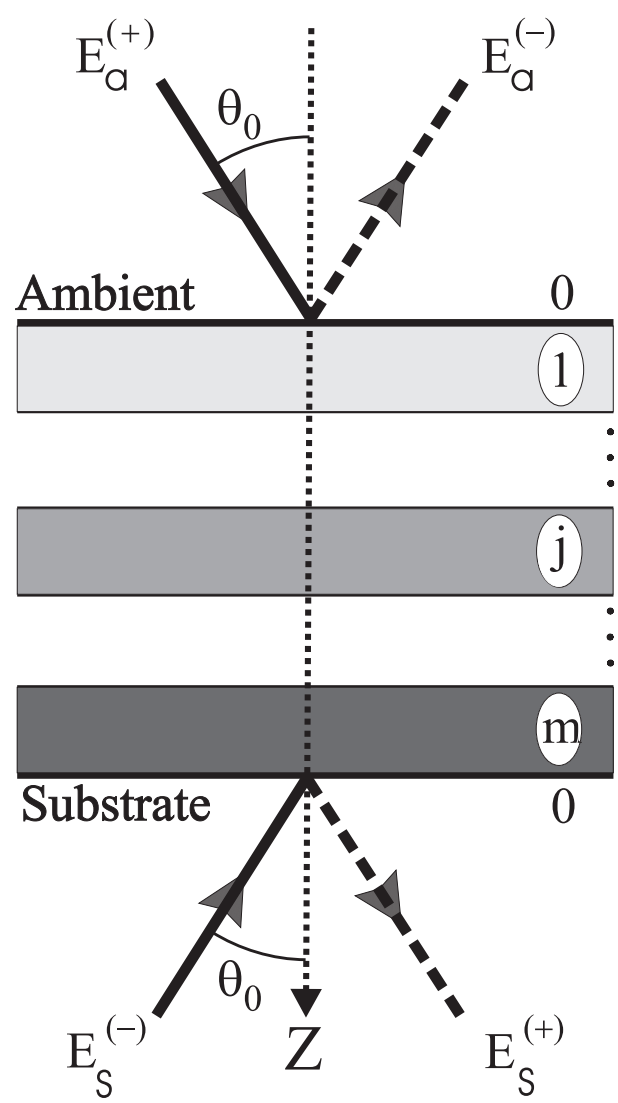

FIG. 1: Wave vectors of the input $\left[E_{a}^{(+)}\right.$and $\left.E_{s}^{(-)}\right]$and output $\left[E_{a}^{(-)}\right.$and $\left.E_{s}^{(+)}\right]$fields in a multilayer sandwiched between two identical semi-infinite ambient and substrate media.

\section{MULTILAYER ACTION IN THE UNIT DISK}

The theory of reflection and transmission of light by stratified planar structures is of wide interest in optics. A considerable amount of theoretical work has been done on this topic, a detailed discussion of which can be found in a number of books [8, 10, 11, 12, 13, 14]. Nowadays, the standard method uses a matrix representation of the field in each medium, as pioneered by Abelès 15. In this paper, we follow the elegant approach developed by Hayfield and White [16].

We deal with a stratified structure that consists of a stack of plane-parallel layers sandwiched between two semi-infinite ambient $(a)$ and substrate $(s)$ media that we shall assume to be identical, in order to simplify as much as possible the calculations. Hereafter all the media are supposed to be lossless, linear, homogeneous, and isotropic. We choose the $Z$ axis perpendicular to the boundaries and directed as in Fig. 1.

A monochromatic linearly polarized plane wave falls from the ambient making an angle $\theta_{0}$ with the normal to the first interface and with an amplitude $E_{a}^{(+)}$. The electric field is either in the plane of incidence ( $p$ po- larization) or perpendicular to the plane of incidence $(s$ polarization). Since the multilayer has two input channels, we consider as well another plane wave of the same frequency and polarization, and with amplitude $E_{s}^{(-)}$, incident from the substrate at the same angle $\theta_{0}$. This guarantees that all the multiple reflected and transmitted waves superpose with the same wave vectors. The overall output fields in the ambient and the substrate will be denoted $E_{a}^{(-)}$and $E_{s}^{(+)}$, respectively. In the common experimental situation, there is no light incident from the substrate $\left[E_{s}^{(-)}=0\right]$, although there are relevant situations in which both input fields are present (e. g., at the beam splitter in a Michelson interferometer).

The field amplitudes at each side of the multilayer are related by the linear relation

$$
\left(\begin{array}{c}
E_{a}^{(+)} \\
E_{a}^{(-)}
\end{array}\right)=\mathbf{M}_{a s}\left(\begin{array}{c}
E_{s}^{(+)} \\
E_{s}^{(-)}
\end{array}\right),
$$

where the multilayer transfer matrix $\mathbf{M}_{a s}$ can be shown to be 14.

$$
\mathbf{M}_{a s}=\left[\begin{array}{cc}
1 / T_{a s} & R_{a s}^{*} / T_{a s}^{*} \\
R_{a s} / T_{a s} & 1 / T_{a s}^{*}
\end{array}\right] \equiv\left[\begin{array}{cc}
\alpha & \beta \\
\beta^{*} & \alpha^{*}
\end{array}\right]
$$

Here the complex numbers $R_{a s}$ and $T_{a s}$, which can be expressed as

$$
R_{a s}=\left|R_{a s}\right| \exp (i \rho), \quad T_{a s}=\left|T_{a s}\right| \exp (i \tau),
$$

are, respectively, the overall reflection and transmission coefficients for a wave incident from the ambient. Because $\left|R_{a s}\right|^{2}+\left|T_{a s}\right|^{2}=1$, we have $\operatorname{det} \mathbf{M}_{a s}=|\alpha|^{2}-|\beta|^{2}=$ 1 , and then $\mathbf{M}_{a s}$ belongs to the group $\mathrm{SU}(1,1)$.

In Ref. 17] we have proposed viewing the multilayer action in a relativisticlike framework, giving a formal equivalence between the fields in Eq. (2.1) and spacetime coordinates in a $(2+1)$-dimensional space. These coordinates verify $\left(x^{0}\right)^{2}-\left(x^{1}\right)^{2}-\left(x^{2}\right)^{2}=1$, which defines a unit two-sheeted hyperboloid characteristic of the group $\mathrm{SO}(2,1)$. If one uses stereographic projection taking the south pole as projection center, the upper sheet of the unit hyperboloid is projected into the unit disk, the lower sheet into the external region, while the infinity goes to the boundary of the unit disk.

The geodesics in the hyperboloid are intersections with the hyperboloid of planes passing through the origin. Consequently, hyperbolic lines are obtained from these by stereographic projection and they correspond to circle arcs that orthogonally cut the boundary of the unit disk.

In many instances (e.g., in polarization optics [13]) we are interested in the transformation properties of field quotients rather than the fields themselves. Therefore, it seems natural to consider the complex numbers

$$
z_{s}=\frac{E_{s}^{(-)}}{E_{s}^{(+)}}, \quad z_{a}=\frac{E_{a}^{(-)}}{E_{a}^{(+)}} .
$$


The action of the multilayer given in Eq. (2.2) can be then seen as a function $z_{a}=f\left(z_{s}\right)$ that can be appropriately called the multilayer transfer function 18].

From a geometrical viewpoint, this function defines a transformation of the complex plane, mapping the point $z_{s}$ into the point $z_{a}$, according to [19]

$$
z_{a}=\Phi\left[\mathrm{M}_{a s}, z_{s}\right]=\frac{\beta^{*}+\alpha^{*} z_{s}}{\alpha+\beta z_{s}}
$$

which is a bilinear or Möbius transformation. One can check that the unit disk, the external region and the unit circle remain invariant under the multilayer action. Note also that, when no light impinges from the substrate $z_{s}=0$ and then $z_{a}=R_{a s}$. It is worth mentioning that this approach is quite general, since it provides the transformed of any point in the unit disk for every values of the input fields $E_{a}^{(+)}$and $E_{s}^{(-)}$.

To classify the multilayer action it proves convenient to work out the fixed points of the mapping; that is, the field configurations such that $z_{a}=z_{s} \equiv z_{f}$ in Eq. [2.5) [20]:

$$
z_{f}=\Phi\left[\mathbf{M}_{a s}, z_{f}\right]
$$

whose solutions are

$$
z_{f}=\frac{1}{2 \beta}\left\{-2 i \operatorname{Im}(\alpha) \pm \sqrt{\left[\operatorname{Tr}\left(\mathbf{M}_{a s}\right)\right]^{2}-4}\right\} .
$$

When $\left[\operatorname{Tr}\left(\mathbf{M}_{a s}\right)\right]^{2}<4$ the multilayer action is elliptic and it has only one fixed point inside the unit disk. Since in the Euclidean geometry a rotation is characterized for having only one invariant point, this multilayer action can be appropriately called a hyperbolic rotation.

When $\left[\operatorname{Tr}\left(\mathbf{M}_{a s}\right)\right]^{2}>4$ the action is hyperbolic and it has two fixed points, both on the boundary of the unit disk. The geodesic line joining these two fixed points remains invariant and thus, by analogy with the Euclidean case, this action will be called a hyperbolic translation.

Finally, when $\left[\operatorname{Tr}\left(\mathbf{M}_{a s}\right)\right]^{2}=4$ the multilayer action is parabolic and it has only one (double) fixed point on the boundary of the unit disk.

Here we will be concerned only with the case $\left[\operatorname{Tr}\left(\mathbf{M}_{a s}\right)\right]^{2}>4$, since it is known that any element of $\mathrm{SU}(1,1)$ can be written (in many ways) as the product of two hyperbolic translations [7]. The axis of the hyperbolic translation is the geodesic line joining the two fixed points. A point on the axis will be translated to another point, a (hyperbolic) distance 21]

$$
\zeta=2 \ln \left(\frac{1}{2}\left\{\operatorname{Tr}\left(\mathbf{M}_{a s}\right)+\sqrt{\left[\operatorname{Tr}\left(\mathbf{M}_{a s}\right)\right]^{2}-4}\right\}\right)
$$

along the axis.

\section{HYPERBOLIC TURNS AND THEIR COMPOSITION}

In Euclidean geometry, a translation of magnitude $\zeta$ along a line $\gamma$ can be seen as the product of two reflections in any two straight lines orthogonal to $\gamma$, separated

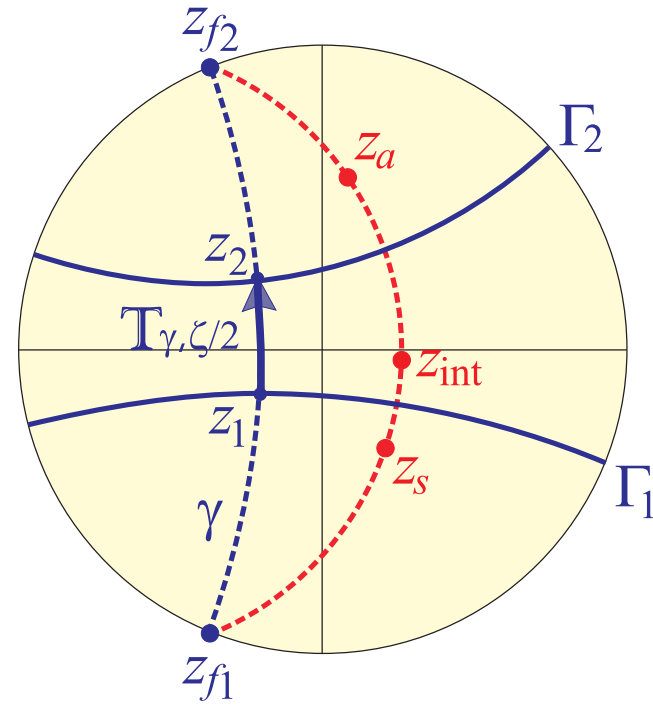

FIG. 2: Representation of the sliding turn $\mathbb{T}_{\gamma, \zeta / 2}$ in terms of two reflections in two lines $\Gamma_{1}$ and $\Gamma_{2}$ orthogonal to the axis of the translation $\gamma$, which has two fixed points $z_{f 1}$ and $z_{f 2}$. The transformation of a typical off axis point $z_{s}$ is also shown.

a distance $\zeta / 2$. This idea can be translated much in the same way to the unit disk, once the concepts of line and distance are understood in the hyperbolic sense. In consequence, any pair of points $z_{1}$ and $z_{2}$ on the axis of the translation $\gamma$ at a distance $\zeta / 2$ can be chosen as intersections of $\Gamma_{1}$ and $\Gamma_{2}$ (orthogonal lines to $\gamma$ ) with $\gamma$. It is then natural to associate to the translation an oriented segment of length $\zeta / 2$ on $\gamma$, but otherwise free to slide on $\gamma$ (see Fig. 2). This is analogous to Hamilton's turns, and will be called a hyperbolic turn $\mathbb{T}_{\gamma, \zeta / 2}$.

Note that using this construction, an off-axis point such as $z_{s}$ will be mapped by these two reflections (through an intermediate point $z_{\text {int }}$ ) to another point $z_{a}$ along a curve equidistant to the axis. These other curves, unlike the axis of translation, are not hyperbolic lines. The essential point is that once the turn is known, the transformation of every point in the unit disk is automatically established.

Alternatively, we can formulate the concept of turn as follows. Let $\mathbf{M}_{a s}$ be a hyperbolic translation with $\operatorname{Tr}\left(\mathbf{M}_{a s}\right)$ positive (equivalently, $\operatorname{Re}(\alpha)>1$ ). Then, $\mathbf{M}_{a s}$ is positive definite and one can ensure that its square root exists and reads as

$$
\sqrt{\mathbf{M}_{a s}}=\frac{1}{\sqrt{2[\operatorname{Re}(\alpha)+1]}}\left[\begin{array}{cc}
\alpha+1 & \beta \\
\beta^{*} & \alpha^{*}+1
\end{array}\right] .
$$

This matrix has the same fixed points as $\mathbf{M}_{a s}$, but the translated distance is just half the induced by $\mathbf{M}_{a s}$; i.e.,

$$
\zeta\left(\mathbf{M}_{a s}\right)=2 \zeta\left(\sqrt{\mathbf{M}_{a s}}\right) .
$$

This suggests that the matrix $\sqrt{\mathbf{M}_{a s}}$ can be appropriately associated to the turn $\mathbb{T}_{\gamma, \zeta / 2}$ that represents the translation induced by $\mathbf{M}_{a s}$. Therefore, we symbolically 


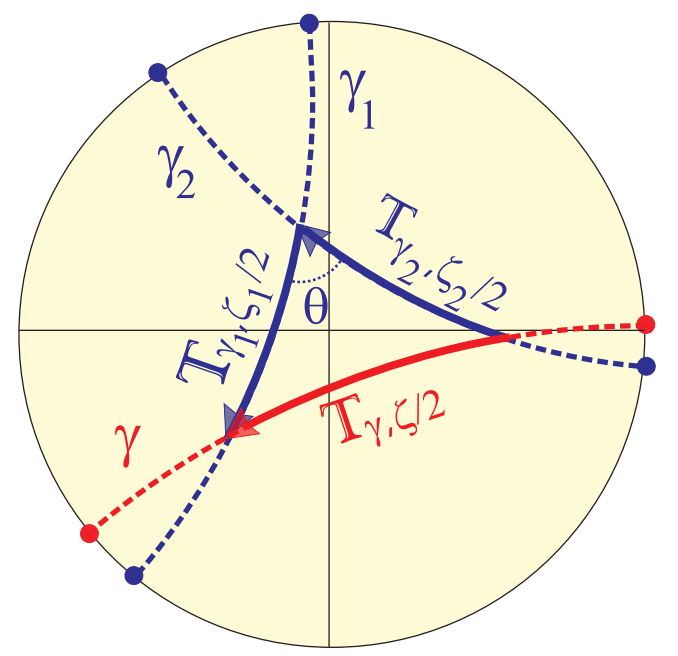

FIG. 3: Composition of two hyperbolic turns $\mathbb{T}_{\gamma_{1}, \zeta_{1} / 2}$ and $\mathbb{T}_{\gamma_{2}, \zeta_{2} / 2}$ by using a parallelogramlike law when the axes $\gamma_{1}$ and $\gamma_{2}$ of the translations intersect.

write

$$
\mathbb{T}_{\gamma, \zeta / 2} \mapsto \sqrt{\mathbf{M}_{a s}}
$$

One may be tempted to extend the Euclidean composition of concurrent vectors to the problem of hyperbolic turns. Indeed, this can be done quite straightforwardly [5]. Let us consider the case of the composition of two of these multilayers represented by the matrices $\mathbf{M}_{1}$ and $\mathbf{M}_{2}$ (for simplicity, we shall henceforth omit the subscript as) of parameters $\zeta_{1}$ and $\zeta_{2}$ along intersecting axes $\gamma_{1}$ and $\gamma_{2}$, respectively. Take the associated turns $\mathbb{T}_{\gamma_{1}, \zeta_{1} / 2}$ and $\mathbb{T}_{\gamma_{2}, \zeta_{2} / 2}$ and slide them along $\gamma_{1}$ and $\gamma_{2}$ until they are "head to tail". Afterwards, the turn determined by the free tail and head is the turn associated to the resultant, which represents thus a translation of parameter $\zeta$ along the line $\gamma$. This construction is shown in Fig. 3, where the noncommutative character is also evident.

In Euclidean geometry, the resultant of this parallelogram law can be quantitatively determined by a direct application of the cosine theorem. For any hyperbolic triangle with sides of lengths $\zeta_{1}$ and $\zeta_{2}$ that make an angle $\theta$, we take the expression from any standard book on hyperbolic geometry [21]

$$
\cosh \zeta=\cosh \zeta_{1} \cosh \zeta_{2}+\sinh \zeta_{1} \sinh \zeta_{2} \cos \theta,
$$

where $\theta$ is the angle between both sides. Moreover, for future use we quote that the (hyperbolic) area $\Omega$ of the geodesic triangle is

$$
\tan (\Omega / 2)=\frac{\tanh \left(\zeta_{1} / 2\right) \tanh \left(\zeta_{2} / 2\right) \sin \theta}{1-\tanh \left(\zeta_{1} / 2\right) \tanh \left(\zeta_{2} / 2\right) \cos \theta}
$$

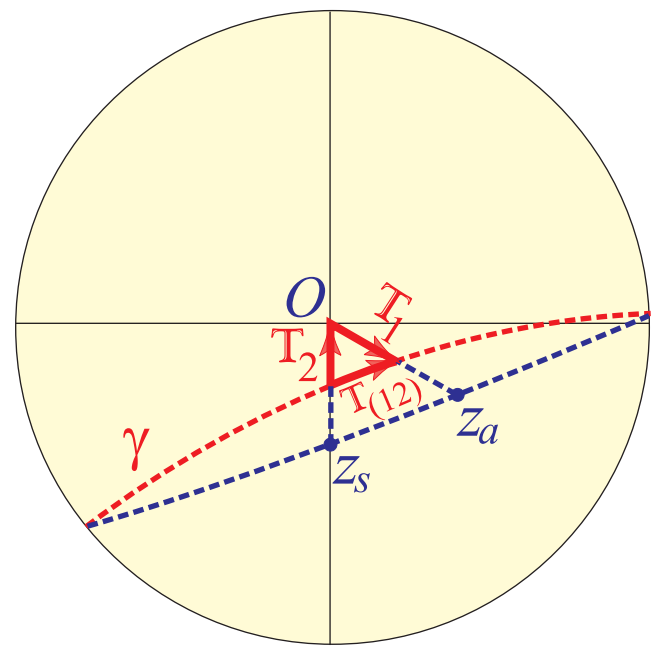

FIG. 4: Composition of two multilayers represented by Hermitian matrices $\mathbf{H}_{1}$ and $\mathbf{H}_{2} . \mathbf{H}_{2}$ maps the point $z_{s}=-R_{2}$ into the origin, while $\mathbf{H}_{1}$ maps the origin into $z_{a}=R_{1}$. We show also the associated turns $\mathbb{T}_{1}$ and $\mathbb{T}_{2}$, as well as the resulting one $\mathbb{T}_{(12)}$ obtained via the parallelogram law. The composite multilayer $\mathbf{H}_{1} \mathbf{H}_{2}$ transforms the point $z_{s}$ into $z_{a}$. The data of the corresponding multilayers are given in the text.

\section{APPLICATION: REVEALING THE WIGNER ANGLE IN THE UNIT DISK}

To show how this formalism can account for the existence of peculiar effects in the composition of multilayers, we address here the question of the Wigner angle in the unit disk and propose a simple optical experiment to determine this angle.

The Wigner angle emerges in the study of the composition of two noncollinear pure boosts in special relativity: the combination of two such successive boosts cannot result in a pure boost, but renders an additional pure rotation, usually known as the Wigner rotation 22] (sometimes the name of Thomas rotation [23, 24] is also used). In other words, boosts are not a group.

To fix the physical background, consider three frames of reference $K, K^{\prime}$ and $K^{\prime \prime}$. Frames $K-K^{\prime}$ and $K^{\prime}-K^{\prime \prime}$ have parallel respective axes. Frame $K^{\prime \prime}$ moves with uniform velocity $\mathbf{v}_{2}$ with respect to $K^{\prime}$, which in turn moves with velocity $\mathbf{v}_{1}$ relative to $K$. The Lorentz transformation that connects $K$ with $K^{\prime \prime}$ is given by the product $\mathbf{L}_{1}\left(\mathbf{v}_{1}\right) \mathbf{L}_{2}\left(\mathbf{v}_{2}\right)$, which can be decomposed as

$$
\mathbf{L}_{1}\left(\mathbf{v}_{1}\right) \mathbf{L}_{2}\left(\mathbf{v}_{2}\right)=\mathbf{L}_{(12)}(\mathbf{v}) \mathbf{R}(\Psi)
$$

where one must be careful in operating just in the same order as written in Eq. (4.1). In words, this means that an observer in $K$ sees the axes of $K^{\prime \prime}$ rotated relative to the observer's own axes by a Wigner rotation described by $\mathbf{R}(\Psi)$. The explicit expression for the axis and angle of this rotation can be found, e.g. in Ref. 22] and will be worked out below from the perspective of the multilayer action in the unit disk. 
First, we observe that any matrix $\mathbf{M} \in \mathrm{SU}(1,1)$ can be expressed in a unique way in the form

$$
\mathbf{M}=\mathbf{H U}
$$

where $\mathbf{H}$ is positive definite Hermitian and $\mathbf{U}$ is unitary. One can check by simple inspection that the explicit form of this (polar) decomposition reads as 25]

$$
\begin{aligned}
\mathbf{M} & =\mathbf{H U}=\left[\begin{array}{cc}
1 /|T| & R^{*} /|T| \\
R /|T| & 1 /|T|
\end{array}\right] \\
& \times\left[\begin{array}{cc}
\exp (-i \tau) & 0 \\
0 & \exp (i \tau)
\end{array}\right] .
\end{aligned}
$$

The component $\mathbf{H}$ is equivalent to a pure boost, while $\mathbf{U}$ is equivalent to a spatial rotation.

It is clear from Eq. (4.3) that $[\operatorname{Tr}(\mathbf{H})]^{2}>4$, so it represents a hyperbolic translation. Moreover, one can check that its associated fixed points are diametrically located on the unit circle and so, the axis of this translation is precisely the diameter joining them. By writing now

$$
R=\tanh (\zeta / 2) \exp (i \rho), \quad T=\operatorname{sech}(\zeta / 2) \exp (i \tau),
$$

one can easily check that the matrix $\mathbf{H}$ in Eq. (4.3) transforms the origin into the complex point $R$, that is,

$$
\Phi[\mathbf{H}, 0]=R, \quad \Phi\left[\mathbf{H}^{-1}, R\right]=0 .
$$

In complete analogy with Eq. (4.1) we compose now two multilayers represented by Hermitian matrices $\mathbf{H}_{1}$ and $\mathbf{H}_{2}$ (that is, with zero transmission phase lag $\tau_{1}=$ $\tau_{2}=0$ ) and we get, after simple calculations [26]

$$
\begin{aligned}
\mathbf{H}_{1} \mathbf{H}_{2} & =\mathbf{H}_{(12)} \mathbf{U}=\left[\begin{array}{cc}
1 /\left|T_{(12)}\right| & R_{(12)}^{*} /\left|T_{(12)}\right| \\
R_{(12)} /\left|T_{(12)}\right| & 1 /\left|T_{(12)}\right|
\end{array}\right] \\
& \times\left[\begin{array}{cc}
\exp (-i \Psi / 2) & 0 \\
0 & \exp (i \Psi / 2)
\end{array}\right]
\end{aligned}
$$

where

$$
\begin{gathered}
R_{(12)}=\frac{R_{1}+R_{2}}{1+R_{1}^{*} R_{2}}, \quad T_{(12)}=\frac{\left|T_{1} T_{2}\right|}{1+R_{1}^{*} R_{2}}, \\
\frac{\Psi}{2}=\arg \left[T_{(12)}\right]=\arg \left(1+R_{1} R_{2}^{*}\right),
\end{gathered}
$$

and the subscripts 1 and 2 refer to the corresponding multilayer. The appearance of an extra unitary matrix in Eq. (4.6) is the signature of a Wigner rotation in the multilayer composition and, accordingly, the Wigner angle $\Psi$ is just twice the phase of the transmission coefficient of the compound multilayer.

To view this Wigner angle in the unit disk, let $z_{s}$ be the point in the substrate that is transformed by the multilayer $\mathbf{H}_{2}$ into the origin, and let $z_{a}$ be the result of transforming the origin by $\mathbf{H}_{1}$. According to Eq. (4.5), one has

$$
\Phi\left[\mathbf{H}_{2},-R_{2}\right]=0, \quad \Phi\left[\mathbf{H}_{1}, 0\right]=R_{1} .
$$

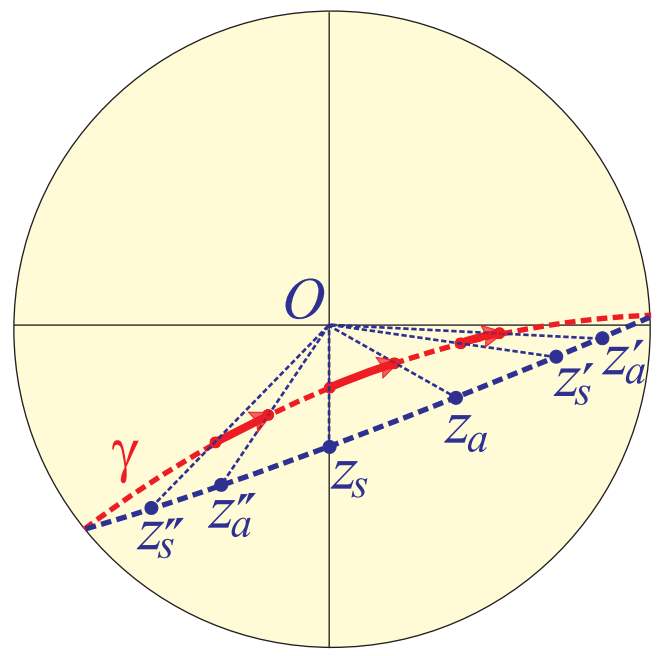

FIG. 5: The same as in Fig. 4 but now the resulting turn $\mathbb{T}_{(12)}$ has been slided to three different positions along the axis. The corresponding points are also transformed by $\mathbf{H}_{1} \mathbf{H}_{2}$. All the geodesic triangles plotted have the same hyperbolic area $\Psi$.

Consider now the (geodesic) triangle defined by the points $z_{s}, O$ and $z_{a}$ in Fig. 4. The general formula (3.5) gives for this triangle

$$
\Omega=\Psi
$$

which confirms the geometric nature of this Wigner angle, since it can be understood in terms of the area (or equivalently, the anholonomy) of a closed circuit [27, 28].

According to the ideas developed in Section 3, we can reduce the multilayers $\mathbf{H}_{1}$ and $\mathbf{H}_{2}$ to the associated turns, represented by arrows in Fig. 4. The "head to tail" rule applied to $\mathbb{T}_{1}$ and $\mathbb{T}_{2}$ (for simplicity, we omit in the subscripts of these turns the corresponding parameters) immediately gives the resulting turn $\mathbb{T}_{(12)}$. However, note that, if we follow the formal prescription shown in Eq. (3.3) and ascribe $\mathbb{T}_{1} \mapsto \sqrt{\mathbf{H}_{1}}$ and $\mathbb{T}_{2} \mapsto \sqrt{\mathbf{H}_{2}}$, we conclude that the composition law imposes

$$
\mathbb{T}_{(12)} \mapsto \sqrt{\mathbf{H}_{1} \mathbf{H}_{2}}
$$

All these results are independent on the position of the turn. In fact, in Fig. 5 we have put the turn $\mathbb{T}_{(12)}$ in different positions along the axis $\gamma$. In every position, we have drawn two radii passing through the head and the tail of $\mathbb{T}_{(12)}$ and taken on them twice the hyperbolic distance from the origin. The pairs of points obtained in this way (such as $z_{s}^{\prime}$ and $z_{a}^{\prime}$ ) are transformed precisely by $\mathbf{H}_{1} \mathbf{H}_{2}$. In other words, $\mathbf{H}_{1} \mathbf{H}_{2}$ can be decomposed in many ways as the composition of two Hermitian matrices and every geodesic triangle $z_{s}^{\prime} O z_{a}^{\prime}$ has the same hyperbolic area $\Psi$.

It seems pertinent to conclude by showing an experimental implementation of the data shown in Fig. 4. To this end, we first recall [13] that for a single plate of refractive index $n_{j}$ and thickness $d_{j}$ imbedded in air 

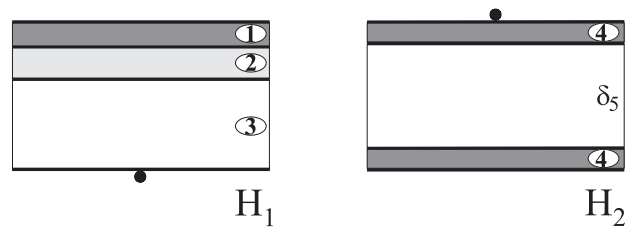

FIG. 6: Scheme of two Hermitian multilayers $\mathbf{H}_{1}$ and $\mathbf{H}_{2}$. The compound multilayer $\mathbf{H}_{1} \mathbf{H}_{2}$ obtained putting together these two components induces a Wigner rotation of angle $2 \tau$.

$\left(n_{0}=1\right)$ illuminated with a monochromatic light of wavelength in vacuo $\lambda$, a standard calculation gives the transfer matrix $\mathbf{M}_{0 j 0}$ with the following reflection and transmission coefficients:

$$
\begin{aligned}
& R_{0 j 0}=\frac{r_{0 j}\left[1-\exp \left(-i 2 \delta_{j}\right)\right]}{1-r_{0 j}^{2} \exp \left(-i 2 \delta_{j}\right)}, \\
& T_{0 j 0}=\frac{t_{0 j} t_{j 0} \exp \left(-i \delta_{j}\right)}{1-r_{0 j}^{2} \exp \left(-i 2 \delta_{j}\right)},
\end{aligned}
$$

where $r_{0 j}$ and $t_{0 j}$ are the Fresnel reflection and transmission coefficients at the interface $0 j$ (which applies to both $p$ and $s$ polarizations by the simple attachment of a subscript $p$ or $s$ ) and $\delta_{j}$ is the plate phase thickness

$$
\delta_{j}=\frac{2 \pi}{\lambda} n_{j} d_{j} \cos \theta_{j},
$$

$\theta_{j}$ being the angle of refraction in the layer. The transfer matrix for the coherent addition of $m$ of these layers is

$$
\mathbf{M}=\prod_{j=1}^{m} \mathbf{M}_{0 j 0} .
$$

As shown in Fig. 6, we take as the first multilayer $\mathbf{H}_{1}$ the lossless system formed by two thin films, one of zinc sulphide (with refractive index $n_{1}=2.3$ and thickness $d_{1}=80 \mathrm{~nm}$ ) and the other of cryolite (with refractive index $n_{2}=1.35$ and thickness $d_{2}=104 \mathrm{~nm}$ ), deposited on a glass substrate (with refractive index $n_{3}=1.5$ and thickness $d_{3}=1.3 \mathrm{~mm}$ ), and imbedded in air. The light has a wavelength in vacuo of $\lambda_{0}=546 \mathrm{~nm}$ and falls from the ambient at normal incidence. Such a simple system could be manufactured with standard evaporation techniques.

We have performed a computer simulation of the performance of this multilayer $\mathbf{H}_{1}$ using a standard package, obtaining $T_{1}=0.9055$ and $R_{1}=0.3736-0.2014 i$, which in turn gives $\tau_{1}=0$ and $\rho_{1}=-0.4944 \mathrm{rad}$.

Our second multilayer $\mathbf{H}_{2}$ is a symmetric system formed by two films of zinc sulphide and thickness $d_{4}=$ $40 \mathrm{~nm}$ separated by a spacer of air with a phase thickness $\delta_{5}=3.707 \mathrm{rad}$. For this subsystem we have $T_{2}=0.9399$ and $R_{2}=0.3413 i$, and therefore $\tau_{2}=0$ and $\rho_{2}=\pi / 2$ rad.

When these two multilayer are put together by the marked points in Fig. 6, the resulting one has a transmission phase lag of $\tau=-0.1361 \mathrm{rad}$, which is just half the area of the geodesic triangle $z_{s} O z_{a}$ in Fig. 4 , as predicted by the theory.

In summary, we expect that the geometrical approach presented here will be an interesting tool for representing in a graphical way the multilayer action. Moreover, the composition law of these turns allows for a clear understanding of the nontrivial effects appearing in the composition of multilayers.

We stress that the benefit of this approach lies not in any inherent advantage in terms of efficiency in solving problems in layered structures. Rather, we expect that turns could provide a general and unifying tool to analyze multilayer performance in an elegant way that, in addition, is closely related to other fields of physics.

\section{Acknowledgments}

We wish to thank José María Montesinos and Mariano Santander for enlightening discussions.

Corresponding author Luis L. Sánchez-Soto e-mail address is lsanchez@fis.ucm.es.
[1] W. R. Hamilton, Lectures on Quaternions (Hodges and Smith, Dublin, 1853).

[2] L. C. Biedenharn and J. D. Louck, Angular Momentum in Quantum Physics (Addison, Reading, MA 1981).

[3] J. J. Monzón and L. L. Sánchez-Soto, "Lossless multilayers and Lorentz transformations: more than an analogy," Opt. Commun. 162, 1-6 (1999).

[4] J. J. Monzón and L. L. Sánchez-Soto, "Fully relativisticlike formulation of multilayer optics," J. Opt. Soc. Am. A 16, 2013-2018 (1999).

[5] M. Juárez and M. Santander, "Turns for the Lorentz group," J. Phys. A 15, 3411-3424 (1982).

[6] R. Simon, N. Mukunda, and E. C. G. Sudarshan, "Hamil- ton's Theory of Turns Generalized to $\operatorname{Sp}(2, \mathrm{R})$," Phys. Rev. Lett. 62, 1331-1334 (1989).

[7] R. Simon, N. Mukunda, and E. C. G. Sudarshan, "The theory of screws: A new geometric representation for the group SU(1, 1)," J. Math. Phys. 30, 1000-1006 (1989).

[8] H. A. Macleod, Thin-film Optical Filters (Adam Hilger, Bristol, UK, 1986).

[9] J. H. Apfel, "Graphics in Optical Coating Design," Appl. Opt. 11, 1303-1312 (1972)

[10] O. S. Heavens, Optical Properties of Thin Solid Films (Dover, New York, 1991).

[11] L. M. Brekovskikh, Waves in Layered Media (Academic, New York, 1960). 
[12] J. Lekner, Theory of Reflection (Dordrecht, The Netherlands, 1987).

[13] R. M. A. Azzam and N. M. Bashara, Ellipsometry and Polarized Light (North-Holland, Amsterdam, 1987).

[14] P. Yeh, Optical Waves in Layered Media (Wiley, New York, 1988)

[15] F. Abelès, "Sur la propagation des ondes electromagnetiques dans les milieux statifiés," Ann. Phys. (Paris) 3, 504-520 (1948)

[16] P. C. S. Hayfield and G. W. T. White, "An assessment of the stability of the Drude-Tronstad polarized light method for the study of film growth on polycrystalline metals," in Ellipsometry in the Measurements of Surfaces and Thin Films, E. Passaglia, R. R. Stromberg, and J. Kruger, eds., Natl. Bur. Stand. Misc. Publ. 256 (U.S. GPO, Washington, D.C., 1964), pp. 157-200.

For a more recent review of the model see Ref. [13], Sec. 4.6.

[17] T. Yonte, J. J. Monzón, L. L. Sánchez-Soto, J. F. Cariñena, and C. López-Lacasta, "Understanding multilayers from a geometrical viewpoint," J. Opt. Soc. Am. A 19, 603-609 (2002).

[18] I. Ohlídal and D. Franta, Ellipsometry of Thin Film Systems, Progress in Optics (Edited by E. Wolf) 41, 181-282 (North-Holland, Amsterdam, 2000).

[19] J. J. Monzón, T. Yonte, L. L. Sánchez-Soto, and J. F.
Cariñena, "Geometrical setting for the classification of multilayers," J. Opt. Soc. Am. A 19, 985-991 (2002).

[20] L. L. Sánchez-Soto, J. J. Monzón, T. Yonte, and J. F. Cariñena, "Simple trace criterion for classification of multilayers," Opt. Lett. 26, 1400-1402 (2001).

[21] A. F. Beardon, The Geometry of Discrete Groups (Springer, New York, 1983) Chap. 7.

[22] A. Ben-Menahem, "Wigner's rotation revisited," Am. J. Phys. 53, 62-66 (1985).

[23] D. A. Jackson, Classical Electrodynamics (Wiley, New York, 1975).

[24] A. A. Ungar, "The relativistic velocity composition paradox and the Thomas rotation," Found. Phys. 19, 13851396 (1989).

[25] J. J. Monzón and L. L. Sánchez-Soto, "Origin of the Thomas rotation that arises in lossless multilayers," J. Opt. Soc. Am. A 16, 2786-2792 (1999).

[26] J. J. Monzón and L. L. Sánchez-Soto, "A simple optical demonstration of geometric phases from multilayer stacks: the Wigner angle as an anholonomy," J. Mod. Opt. 48, 21-34 (2001).

[27] A. Shapere and F. Wilczek (Editors), Geometric Phases in Physics (World Scientific, Singapore, 1989)

[28] P. K. Aravind, "The Wigner angle as an anholonomy in rapidity space," Am. J. Phys. 65, 634-636 (1997). 